УДК 681.5.08

\author{
Д.В. Павлов \\ Новгородский государственный университет \\ имени Ярослава Мудрого, Великий Новгород, Россия \\ МАТЕМАТИЧЕСКАЯ МОДЕЛЬ \\ ВОЛОКОННО-ОПТИЧЕСКОГО ГИРОСКОПА \\ ДЛЯ СТАТИЧЕСКОЙ СИСТЕМЫ
}

\begin{abstract}
Кратко описана математическая модель волоконно-оптического гироскопа VG910Q. Для разработки эффективной математической модели используется среда Simulink, входящая в состав MatLab. B имитационном моделировании используются виртуальные модули и устройства, поведенческая модель которых максимально приближена к реальной физической модели.
\end{abstract}

Ключевые слова: имитационная модель, волоконно-оптический гироскоп, стабилизация.

\author{
D.V. Pavlov \\ Yaroslav-the-Wise Novgorod State University, \\ Velikii Novgorod, Russian Federation

\section{MATHEMATICAL MODEL OF A FIBER-OPTICAL GYROSCOPE FOR STATIC SYSTEM}

In this work the mathematical model of a fiber-optical gyroscope of VG910Q is briefly described. For development of effective mathematical model the Simulink environment which is a part of MatLab is used. In imitating modeling virtual modules and devices which behavioural model is most approached to real physical model are used.

Keywords: imitating model, fiber-optical gyroscope, stabilization.

В оптико-электронных системах слежения и наведения, использующихся на борту истребителей, требуется стабилизация визирной оси. Для повышения точности стабилизации необходимо усовершенствование модуля, который выполняет задачу стабилизации. Модернизация данного модуля может заключаться:

- в изменении элементной базы изделия;

- усовершенствовании программно-алгоритмического обеспечения.

Изменение элементной базы приведет к изменению габаритов модуля. Однако изменять габаритные характеристики и элементную 
базу модуля не всегда представляется возможным из-за жестких требований к массо-габаритным характеристикам. Поэтому повышения точности стабилизации визирной оси можно достичь путем усовершенствования алгоритмов. В частности, точный учет поведенческой модели датчиков, входящих в состав модуля стабилизации, позволяет построить математическую модель. Эта модель позволит учесть возможные погрешности датчиков, тем самым скорректировать их.

В данной статье изучается инерциальный измерительный модуль (ИИМ), который входит в состав системы гиростабилизации бортового оборудования оптико-электронного прибора (ОЭП). ОЭП представляет собой бортовое устройство военного истребителя СУ-35. Блок-схема ИИМ представлена на рис. 1.
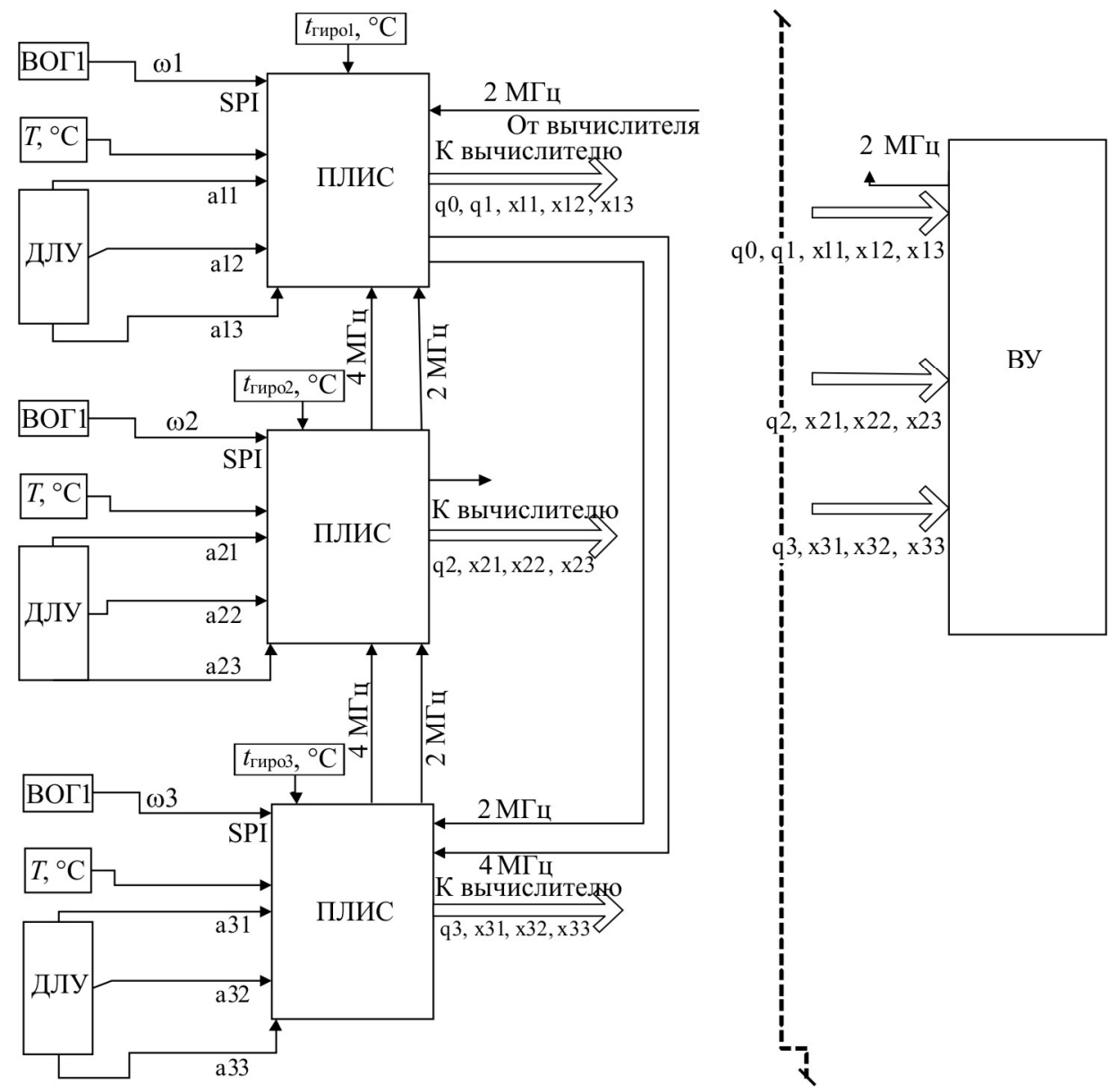

Рис. 1. Блок-схема ИИМ с вычислительным устройством (ВУ) 
ИИМ представляет собой устройство, состоящее из трех отдельных подмодулей. Основные элементы: акселерометр - датчик линейного ускорения (ДЛУ), волоконно-оптический гироскоп (ВОГ), цифровой датчик температуры $\left(T,{ }^{\circ} \mathrm{C}\right)$. В процессорную часть схемы ИИМ входит ПЛИС Altera Cyclone III. ВУ используется как отдельное устройство, на котором выполняются все вычислительные операции с плавающей запятой.

В данной работе кратко описана математическая модель [1] волоконно-оптического гироскопа VG910Q, который используется в ИИМ. Для разработки эффективной математической модели использовалась среда Simulink, входящая в состав MatLab. В имитационном моделировании используются виртуальные модули и устройства, поведенческая модель которых максимально приближена к реальной физической модели.

\section{1. Разработка математической модели волоконно-оптического гироскопа}

В данной работе в качестве объекта исследования была выбрана модель волоконно-оптического гироскопа ВГ910Q («Физоптика»), представленная на рис. 2.

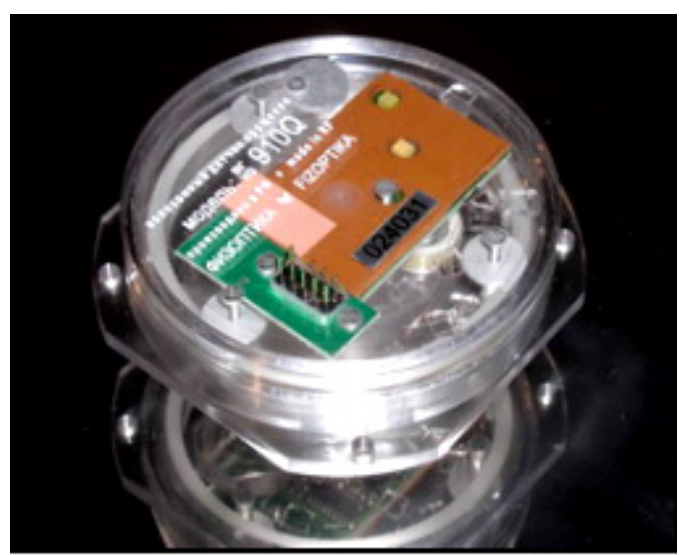

Рис. 2. Волоконно-оптический гироскоп модели ВГ910Q

Ниже приведены технические характеристики гироскопа ВГ910Q. 


\begin{tabular}{|c|c|}
\hline Параметр & Типовые значения \\
\hline Диапазон измеряемых скоростей & 150 град/с \\
\hline Масштабный коэффициент (МК) $k$ & 7 мВ/град/с \\
\hline Рабочая полоса частот & $0-0,45$ кГц \\
\hline Угловой случайный дрейф & 0,04 град/ $\sqrt{4}$ \\
\hline Дрейф нуля (1 б) & 10 град/ч \\
\hline Стабильность МК, $1 \sigma$ & $0,1 \%$ \\
\hline
\end{tabular}

Устройство ВОГ показано на рис. 3. ВОГ является оптическим интерферометрическим датчиком вращения. Это датчик, в котором разность фаз интерферирующих волн определяется внешним физическим эффектом. Иногда волоконно-оптические датчики вращения называют гироскопами. Однако принцип их функционирования не имеет ничего общего с классическим гироскопическим эффектом, обусловленным силой Кориолиса. Принцип работы волоконно-оптического датчика вращения основан на релятивистском эффекте Саньяка - появление фазового сдвига встречных электромагнитных волн во вращающемся кольцевом интерферометре. Корректная модель эффекта Саньяка может быть построена только в рамках Общей теории относительности.

Используемое в датчике оптическое волокно, сохраняющее поляризацию излучения, устраняет эффекты вращения поля и обеспечивает исключительную стабильность и точность измерения угловой скорости.

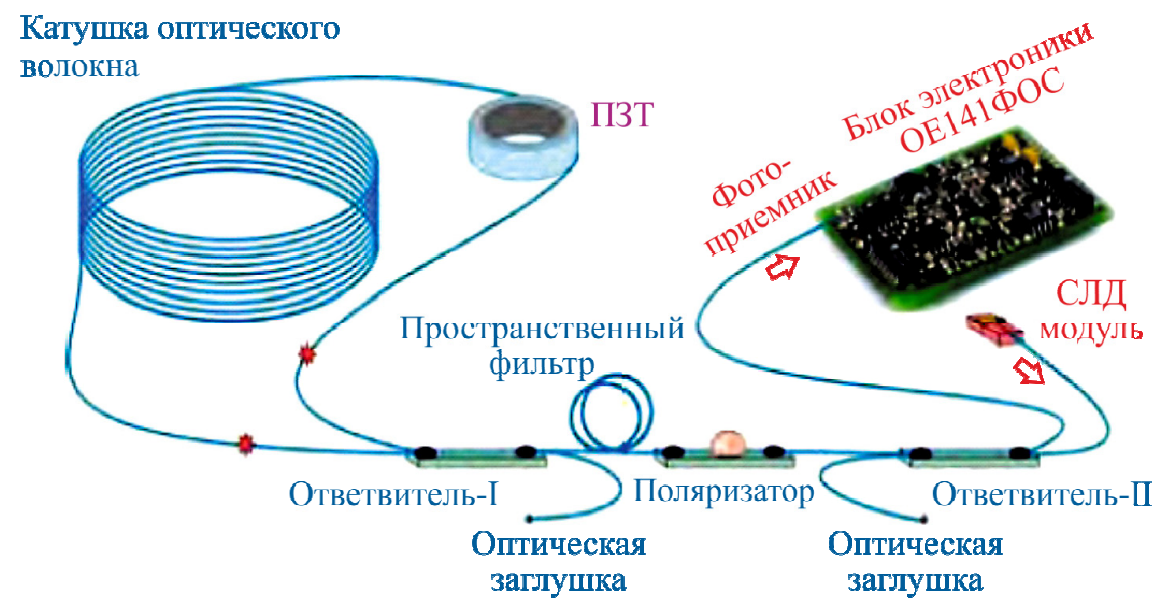

Рис. 3. Устройство волоконно-оптического гироскопа модели ВГ910Q 
Выходной сигнал гироскопа содержит мультипликативную и аддитивную погрешности: мультипликативная связана с искажениями масштабного коэффициента, обусловлеными температурными факторами; аддитивная - с наличием двух факторов, чувствительных к температуре: сигналом дрейфа нуля и шума [2].

Выходной сигнал гироскопа в общем виде можно представить следующим образом:

$$
U_{\text {out }}=k(T) \cdot \omega+U_{d}(T)+N(T),
$$

где $U_{\text {out }}$ - выходное напряжение гироскопа, $\mathrm{B} ; U_{d}(T)$ - дрейф нулевого значения выходного сигнала, $\mathrm{B} ; k(T)$ - масштабный коэффициент гироскопа, В/град/с; $\omega-$ измеряемая угловая скорость, град/с; $N(T)-$ шум гироскопа, В.

Для статической системы $\omega=0$.

Для получения количественной оценки параметров температурной зависимости шума гироскопа и дрейфа нуля были проведены экспериментальные исследования выходного сигнала гироскопа от температуры.

Для моделирования эффекта температурной зависимости дрейфа нуля использована аппроксимация среднего значения выходного сигнала гироскопа $U_{\text {out }}$ полиномом второй степени. Ниже приведены аппроксимирующие полиномы для выборки трех гироскопов модели ВГ910Q:

$$
\begin{aligned}
& U_{d 1}(T)=1,023 \cdot 10^{-7} \cdot T^{2}+2,616 \cdot 10^{-6} \cdot T+0,000307 ; \\
& U_{d 2}(T)=0,853 \cdot 10^{-7} \cdot T^{2}-7,483 \cdot 10^{-6} \cdot T-0,000002 ; \\
& U_{d 3}(T)=3,049 \cdot 10^{-7} \cdot T^{2}+3,178 \cdot 10^{-6} \cdot T+0,000071 .
\end{aligned}
$$

Для получения аппроксимирующих температурных зависимостей СКО были выполнены эксперименты для выборки из трех гироскопов в диапазоне температур от 25 до $48{ }^{\circ} \mathrm{C}$. Получены следующие результаты:

$$
\begin{aligned}
& \sigma_{1}(T)=4,26 \cdot 10^{-6} \cdot T+0,000012 ; \\
& \sigma_{2}(T)=1,77 \cdot 10^{-6} \cdot T+0,000037 \\
& \sigma_{3}(T)=2,45 \cdot 10^{-6} \cdot T+0,000061 .
\end{aligned}
$$

В формулах (2) и (3) параметр $T$ - температура, ${ }^{\circ} \mathrm{C}$.

На базе приведенного алгоритма средствами Simulink [3] разработана имитационная модель ВОГ, представленная на рис. 4. 


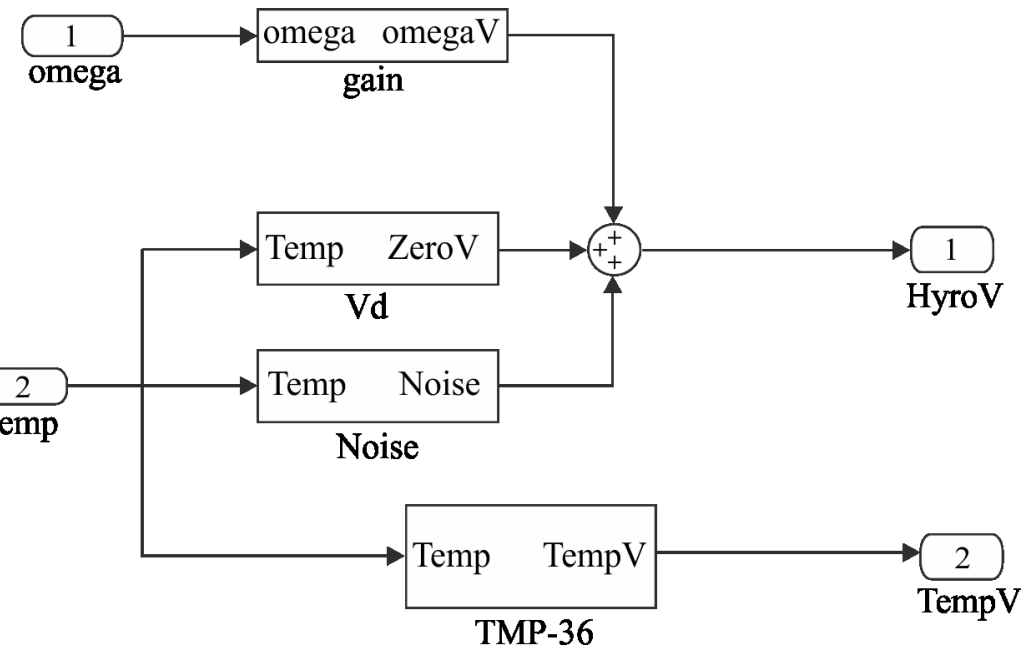

Рис. 4. Блок-диаграмма имитационной модели гироскопа: omega, temp входные порты модели; HyroV, TempV - выходные порты модели; gain - блок, реализующий масштабный коэффициент;

TMP-36 - температурный датчик (градуировочная шкала)

На рис. 5 показано поведение выходных сигналов ВОГа ВГ910Q на интервале температур от +25 до $+48{ }^{\circ} \mathrm{C}$. На рис. 5 показано экспериментальное поведение выходного сигнала ВОГ без использования коррекции с применением его модели (см. рис. 4). На рис. 6 показано экспериментальное поведение выходного сигнала ВОГ с применением коррекции с помощью его модели.

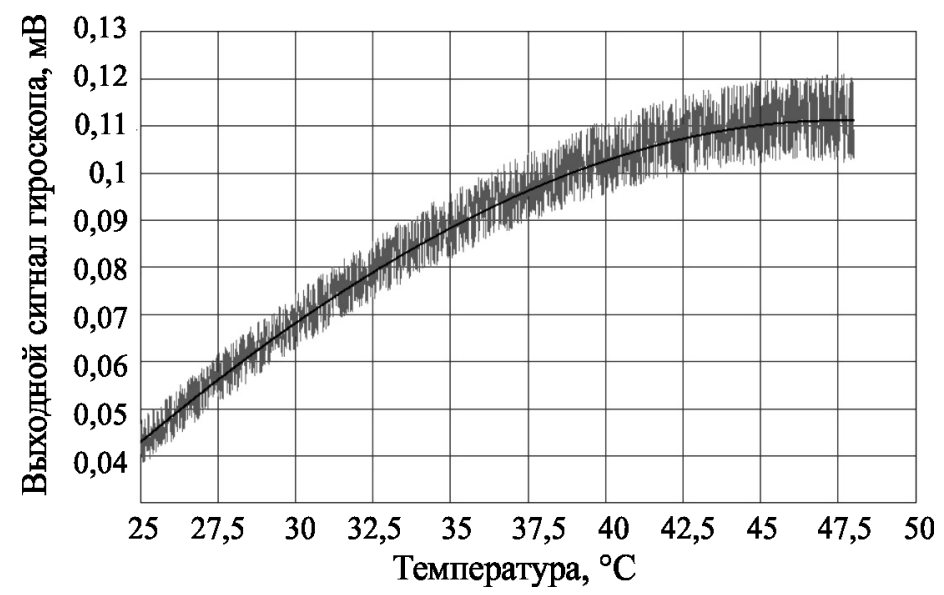

Рис. 5. Зависимость выходного сигнала реального ВОГа ВГ910Q от температуры без коррекции сигналом его математической модели 


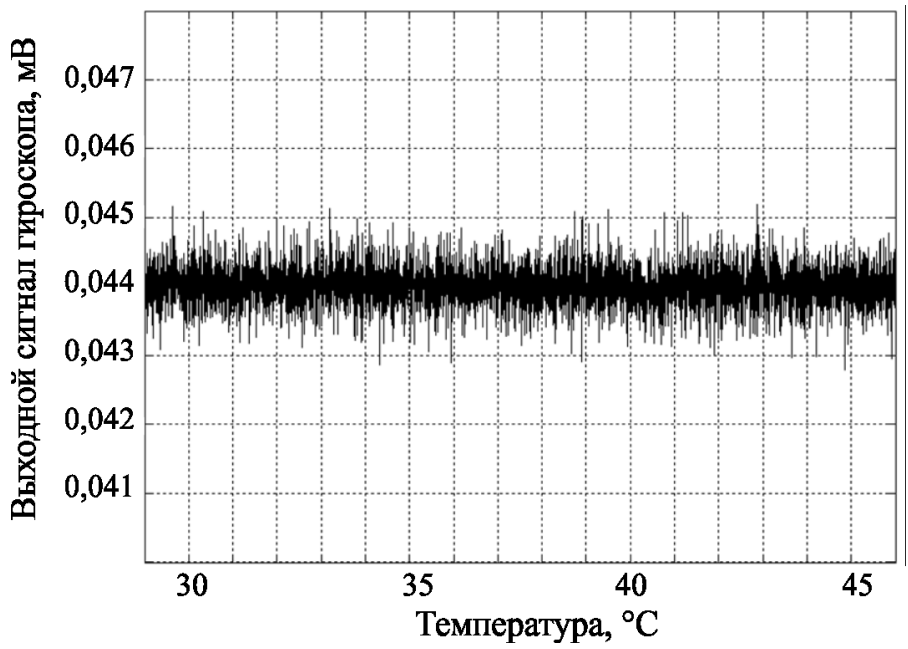

Рис. 6. Зависимость выходного сигнала реального ВОГа ВГ910Q от температуры с коррекцией сигналом его математической модели

На рис. 5 кривая с темной линией показывает среднее значение выходного сигнала реального ВОГ. Более светлые линии (серый цвет) показывают СКО шумового сигнала по уровню $1 \sigma$.

На рис. 6 показан выходной сигнал ВОГ, скорректированный сигналом его математической модели. Из рисунка видно, что температурный дрейф реального ВОГ, который наблюдался на рис. 5, полностью скорректирован. Наличие шумового сигнала с разбросом напряжений от 0,043 до 0,045 мВ связано с остаточными шумами, которые имеют более сложную температурную зависимость и распределение.

При дальнейшей работе ИИМ на борту военного самолета имитационная модель используется для температурной коррекции выходного сигнала с целью повышения стабильности выходных сигналов ИИМ в заданном температурном диапазоне.

Данная имитационная модель учитывает зависимость дрейфа нуля и СКО шума от температуры, что позволяет повысить точность гиростабилизации ОЭП в широком интервале температур на $30 \%$.

\section{Список литературы}

1. Колесов Ю.Б., Сениченков Ю.Б. Визуальное моделирование. СПб.: Профессионал, 2000. - 241 с. 
2. Евстифеева О.М., Игнатьев С.В. Анализ экспериментальных данных для оценки характеристик точности волоконно-оптического гироскопа / ГНЦ РФ ЦНИИ «Электроприбор». - СПб., 1978.

3. Simulink - Simulation and Model-Based Design. - URL: http:// www.mathworks.com/products/simulink.

\section{References}

1. Kolesov Iu.B., Senichenkov Iu.B. Vizual'noe modelirovanie [Visual modeling]. Saint-Petersburg: Professional, 2000, 241 p.

2. Evstifeeva O.M., Ignat'ev S.V. Analiz eksperimental'nykh dannykh dlia otsenki tochnosti volokonno-opticheskogo giroskopa [The analysis of experimental data for an assessment of characteristics of accuracy of a fiberoptical gyroscope]. Saint-Petersburg, 1978.

3. Simulink - Simulation and Model-Based Design, available at: http://www.mathworks.com/products/simulink.

Получено 27.11.2014 